\title{
Pacific
}

Journal of

Mathematics

\section{MINIMAL SURFACES IN $S^{3}$ FOLIATED BY CIRCLES}

Nikolai Kutev and Velichka Milousheva 


\title{
MINIMAL SURFACES IN $S^{3}$ FOLIATED BY CIRCLES
}

\author{
Nikolai Kutev and VelichKa Milousheva
}

\begin{abstract}
We study minimal surfaces in the unit sphere $S^{3}$ that are one-parameter families of circles. Minimal surfaces in $\mathbb{R}^{3}$ foliated by circles were first investigated by Riemann, and a hundred years later Lawson constructed examples of such surfaces in $S^{3}$. We prove that in $S^{3}$ only two types of minimal surfaces are foliated by circles crossing the principal lines at a constant angle. The first type of surfaces are foliated by great circles that are bisectrices of the principal lines, and we show that these are the examples of Lawson. The second type, which are new in the literature, are families of small circles, and the circles are principal lines. We give a constructive formula for these surfaces and an application to the theory of minimal foliated semisymmetric hypersurfaces in $\mathbb{R}^{4}$.
\end{abstract}

\section{Introduction}

We study minimal surfaces in the unit sphere $S^{3}$ in the four-dimensional Euclidean space $\mathbb{R}^{4}$, equipped with the standard Euclidean metric $\langle\cdot, \cdot\rangle$. A surface $M^{2}$ in $S^{3}$ is given by a unit vector-valued function $l(u, v)$ in $\mathbb{R}^{4}$ defined in a domain $\mathscr{D} \subset \mathbb{R}^{2}$, that is,

$$
l(u, v)=\left(l^{1}(u, v), l^{2}(u, v), l^{3}(u, v), l^{4}(u, v)\right) \text { for }(u, v) \in \mathscr{D},
$$

where $\langle l(u, v), l(u, v)\rangle=1$ for $(u, v) \in \mathscr{D}$. Since our considerations are local, we assume that the parameters $(u, v)$ are isothermal (conformal), which means that $\left\langle l_{u}, l_{u}\right\rangle=\left\langle l_{v}, l_{v}\right\rangle$ and $\left\langle l_{u}, l_{v}\right\rangle=0$.

The minimal surfaces in $S^{3}$ are determined by the solutions $l=l(u, v)$ of the system

$$
\begin{aligned}
\Delta l+|\nabla l|^{2} l & =0, \\
\left\langle l_{u}, l_{u}\right\rangle=\left\langle l_{v}, l_{v}\right\rangle, \quad\left\langle l_{u}, l_{v}\right\rangle & =0, \quad\langle l, l\rangle=1,
\end{aligned}
$$

MSC2000: primary 53A10, 35J60; secondary 53A07, 35A07.

Keywords: minimal surfaces in $S^{3}$, Clifford torus, Lawson torus, harmonic map, foliated semisymmetric hypersurface, minimal hypersurface, nonlinear elliptic system.

Milousheva is partially supported by the L. Karavelov Higher School of Civil Engineering, Sofia, Bulgaria, under contract number 10/2009. 
where $\nabla$ and $\Delta$ denote the gradient and the Laplacian operators, respectively, with respect to the Euclidean metric in $\mathbb{R}^{4}$.

The system (1-1) is an Euler-Lagrange system of harmonic maps and has been studied intensively in the last decades by variational methods [Giaquinta et al. 1998a, 1998b; Hildebrandt 1982; Jost 1984, 1991; Struwe 1988].

Our aim is to find the minimal surfaces in $S^{3}$ that satisfy the geometric property that locally they are one-parameter families of circles. Variational methods cannot be applied for studying the geometric structure of these minimal surfaces. Hence, we use a method based on the differential geometry of surfaces in $\mathbb{R}^{4}$ rather than one based on PDEs.

It is well known that the only minimal rotational surface in $\mathbb{R}^{3}$ is the catenoid, which is a surface fibered by circles in parallel planes. Another class of minimal surfaces in $\mathbb{R}^{3}$ foliated by circles in parallel planes consists of the Riemann examples [Riemann 1868]. Enneper [1869] proved that catenoids and Riemann examples are the only minimal surfaces in $\mathbb{R}^{3}$ foliated by circles. A surface in $\mathbb{R}^{3}$ that is determined by a smooth one-parameter family of circles is also called a cyclic surface. Cyclic surfaces of constant mean curvature and cyclic surfaces of constant Gauss curvature in $\mathbb{R}^{3}$ are described in [Nitsche 1989] and [López 2001].

Our idea to find the minimal surfaces in $S^{3}$ that are one-parameter families of circles is motivated by what happens for cyclic minimal surfaces in $\mathbb{R}^{3}$.

A well-known example of a minimal surface in $S^{3}$ is the Clifford torus (the standard flat torus), which consists of two orthogonal families of circles. This is generalized by the Lawson tori [1970], which have two orthogonal families of parametric lines: one of them consists of circles, the other of curves with constant Frenet curvatures in $\mathbb{R}^{4}$.

The circles on the Lawson torus cross the principal lines at an angle $\pi / 4$. Here we will find all minimal surfaces in $S^{3}$ that are one-parameter families of circles crossing the principal lines at a constant angle. We call these surfaces generalized tori. In Theorem 2.2, we prove that there are only two types of generalized tori in $S^{3}$ : The generalized tori of first type are those whose circles are bisectrices of the principal lines. The generalized tori of second type are those whose circles are principal lines. In Theorem 2.3, we show that all generalized tori of first type are Lawson tori. In Theorem 2.4, we give a constructive formula for the generalized tori of second type.

In Section 3, we will point out the relationship between the theory of minimal surfaces in $S^{3}$ and the theory of minimal foliated semisymmetric hypersurfaces in $\mathbb{R}^{4}$. Each minimal surface in $S^{3}$ generates a minimal foliated semisymmetric hypersurface in $\mathbb{R}^{4}$ according to a special construction given in [Ganchev and Milousheva 2007a]. We will illustrate how this construction can be applied to two examples of minimal surfaces in $S^{3}$ to obtain first and second type helicoids, which 
are special minimal foliated semisymmetric hypersurfaces. We will also apply the construction to the class of generalized tori of second type, and thus obtain new minimal foliated semisymmetric hypersurfaces in $\mathbb{R}^{4}$.

\section{Generalized tori in $S^{3}$}

Let $M^{2}: l=l(u, v)$ for $(u, v) \in \mathscr{D}$ be a surface, parametrized by isothermal parameters and lying on the unit sphere $S^{3}$ in $\mathbb{R}^{4}$. In other words, the vector-valued function $l(u, v)$ satisfies the equalities

$$
\begin{aligned}
\left\langle l_{u}, l_{u}\right\rangle & =\left\langle l_{v}, l_{v}\right\rangle=E(u, v), \\
\left\langle l_{u}, l_{v}\right\rangle & =0, \\
\langle l, l\rangle & =1 .
\end{aligned}
$$

Since $l, l_{u}$, and $l_{v}$ are mutually orthogonal, there exists a unique (up to a sign) unit vector field $n(u, v)$ such that $\left\{l, l_{u}, l_{v}, n\right\}$ form an orthogonal basis in $\mathbb{R}^{4}$. Differentiating the equalities (2-1), we get the derivative formulas

$$
\begin{aligned}
l_{u u} & =\frac{E_{u}}{2 E} l_{u}-\frac{E_{v}}{2 E} l_{v}-E l+a_{11} n, \\
l_{u v} & =\frac{E_{v}}{2 E} l_{u}+\frac{E_{u}}{2 E} l_{v}+a_{12} n, \\
l_{v v} & =-\frac{E_{u}}{2 E} l_{u}+\frac{E_{v}}{2 E} l_{v}-E l+a_{22} n,
\end{aligned}
$$

where $a_{i j}(u, v)$ for $i, j=1,2$ are functions defined in $\mathscr{D}$. Hence

$$
l_{u u}+l_{v v}+2 E l=\left(a_{11}+a_{22}\right) n .
$$

$M^{2}$ is a minimal surfaces in $S^{3}$ if and only if $a_{11}+a_{22}=0$. Equation (2-2) implies that $M^{2}$ is minimal if and only if

$$
l_{u u}+l_{v v}+2 E l=0 .
$$

Consequently, the problem of finding the minimal surfaces in $S^{3}$ is equivalent to the solvability of the system

$$
\left\{\begin{array}{l}
\Delta l(u, v)+2 E(u, v) l(u, v)=0, \\
\left\langle l_{u}, l_{u}\right\rangle=\left\langle l_{v}, l_{v}\right\rangle=E(u, v), \quad\left\langle l_{u}, l_{v}\right\rangle=0, \quad\langle l, l\rangle=1
\end{array}\right.
$$

with an appropriate $\mathscr{C}^{\infty}$ smooth scalar function $E(u, v)>0$ in a small neighborhood $\mathscr{D}$ of the origin.

According to the theorem of Hélein [Giaquinta et al. 1998b, page 346], the solutions of system (1-1) (and hence of (2-3)) are $\mathscr{C}^{\infty}$ smooth because $\mathscr{D}$ is a two-dimensional domain. 
Let $M^{2}: l=l(u, v)$ be a minimal surface in $S^{3}$. Then the derivative formulas of $M^{2}$ are

$$
\begin{aligned}
l_{u u} & =\frac{E_{u}}{2 E} l_{u}-\frac{E_{v}}{2 E} l_{v}-E l+a n, \\
l_{u v} & =\frac{E_{v}}{2 E} l_{u}+\frac{E_{u}}{2 E} l_{v}+b n, \\
l_{v v} & =-\frac{E_{u}}{2 E} l_{u}+\frac{E_{v}}{2 E} l_{v}-E l-a n,
\end{aligned}
$$

where $a(u, v)$ and $b(u, v)$ are functions in $\mathscr{D}$. The derivatives $n_{u}$ and $n_{v}$ of $n(u, v)$ satisfy

$$
n_{u}=-\frac{a}{E} l_{u}-\frac{b}{E} l_{v} \quad \text { and } \quad n_{v}=-\frac{b}{E} l_{u}+\frac{a}{E} l_{v} .
$$

Using the Gauss and Codazzi equations (or equivalently, the identities of the mixed third derivatives of $l(u, v)$ and mixed second derivatives of $n(u, v))$ from (2-4) and (2-5), we see that the functions $a(u, v)$ and $b(u, v)$ are harmonic and satisfy the Cauchy-Riemann conditions

$$
b_{u}(u, v)=a_{v}(u, v) \quad \text { and } \quad b_{v}(u, v)=-a_{u}(u, v) .
$$

The Gauss and Codazzi equations for $M^{2}$ also imply the identity

$$
a^{2}+b^{2}=\frac{1}{2} \Delta E-\frac{E_{u}^{2}+E_{v}^{2}}{2 E}+E^{2} .
$$

The Gauss curvature $K$ of $M^{2}$ is given by

$$
K=\frac{E_{u}^{2}+E_{v}^{2}}{2 E^{3}}-\frac{\Delta E}{2 E^{2}}=-\frac{1}{2 E} \Delta \ln E .
$$

Hence, equalities (2-6) and (2-7) imply that the Gauss curvature $K$ is expressed in terms of the functions $a$ and $b$ as

$$
K=1-\frac{a^{2}+b^{2}}{E^{2}} .
$$

The simplest case in which problem (2-3) can be solved completely is the case $K=$ const. Lawson [1969] proved that if $M^{2}$ is a minimal surface in $S^{3}$ of constant Gauss curvature $K$, then either $K=1$ and $M^{2}$ is totally geodesic, or $K=0$ and $M^{2}$ is an open piece of the Clifford torus.

From (2-8) it follows that the case $M^{2}$ is totally geodesic in $S^{3}$ (that is, $M^{2}$ is a sphere with radius 1 ) corresponds to $a=b=0$. Further on we shall consider only the case $(a, b) \neq(0,0)$.

Let us recall that the Clifford torus is a surface in $\mathbb{R}^{4}$ parametrized as

$$
\mathcal{M}: l(u, v)=(\cos u \cos v ; \cos u \sin v ; \sin u \cos v ; \sin u \sin v) .
$$


A direct computation shows that $l(u, v)$ satisfies the equality $l_{u u}+l_{v v}+2 l=0$, and $\left\langle l_{u}, l_{u}\right\rangle=\left\langle l_{v}, l_{v}\right\rangle=1,\left\langle l_{u}, l_{v}\right\rangle=0$ and $\langle l, l\rangle=1$. The parametric lines $u=\mathrm{const}$ and $v=$ const of $\mu$ are circles.

H. B. Lawson [1970] found other minimal surfaces in $S^{3}$ that generalize the Clifford torus: He proved that every "ruled" minimal surface in $S^{3}$ is an open submanifold of one of the surfaces $\mathcal{M}_{\alpha}$ given by

$$
M_{\alpha}: l(x, y)=(\cos x \cos \alpha y ; \cos x \sin \alpha y ; \sin x \cos y ; \sin x \sin y)
$$

for some constant $\alpha>0$. By "ruled", Clifford means it belongs to a one-parameter family of great circles in $S^{3}$. The surface $M_{1}$ is the Clifford torus, and it is the only surface $M_{\alpha}$ with constant Gauss curvature [Lawson 1969]. We call the surfaces $M_{\alpha}$ with $\alpha \neq 1$ Lawson tori.

The tangent space of $\mu_{\alpha}$ is spanned by the vector fields

$$
\begin{aligned}
& l_{x}(x, y)=(-\sin x \cos \alpha y ;-\sin x \sin \alpha y ; \cos x \cos y ; \cos x \sin y), \\
& l_{y}(x, y)=(-\alpha \cos x \sin \alpha y ; \alpha \cos x \cos \alpha y ;-\sin x \sin y ; \sin x \cos y),
\end{aligned}
$$

and the coefficients $E, F$, and $G$ of the first fundamental form of $\mu_{\alpha}$ are given by $E=1, F=0$ and $G=G(x)=\alpha^{2} \cos ^{2} x+\sin ^{2} x$.

Using (2-9) and (2-10), we find the unit normal vector field $n(u, v)$ of $\mathcal{M}_{\alpha}$ that is orthogonal to $\left\{l, l_{u}, l_{v}\right\}$ :

$$
n(x, y)=\frac{(\sin x \sin \alpha y ;-\sin x \cos \alpha y ;-\alpha \cos x \sin y ; \alpha \cos x \cos y)}{\sqrt{\alpha^{2} \cos ^{2} x+\sin ^{2} x}} .
$$

A direct computation shows that the Gauss curvature of $\mu_{\alpha}$ is given by

$$
K=1-\frac{\alpha^{2}}{\left(\alpha^{2} \cos ^{2} x+\sin ^{2} x\right)^{2}},
$$

and obviously $K \neq$ const when $\alpha \neq 1$.

Let us consider the Lawson torus $M_{\alpha}$ for $\alpha \neq 1$. In such case the parametric lines $y=y_{0}=$ const of $\mu_{\alpha}$ are circles, while the parametric lines $x=x_{0}=$ const are curves in $\mathbb{R}^{4}$ with constant Frenet curvatures.

The parametrization (2-9) of $\mathcal{M}_{\alpha}$ is not isothermal. We shall find isothermal parameters for $\mathcal{M}_{\alpha}$ that are also principal parameters of the surface. Consider the change of parameters

$$
\bar{u}=\int_{0}^{x} \frac{1}{\sqrt{G(\tau)}} d \tau \quad \text { and } \quad \bar{v}=v .
$$

Then we obtain $\bar{E}=\bar{G}=G(x(\bar{u}))$ and $\bar{F}=0$, that is, the parameters $(\bar{u}, \bar{v})$ are isothermal. A direct computation shows that the vector function $l(\bar{u}, \bar{v})$ satisfies 
the system

$$
l_{\bar{u} \bar{u}}=\frac{\bar{E}_{\bar{u}}}{2 \bar{E}} l_{\bar{u}}-\bar{E} l, \quad l_{\bar{u} \bar{v}}=\frac{\bar{E}_{\bar{u}}}{2 \bar{E}} l_{\bar{v}}+\alpha n, \quad l_{\bar{v} \bar{v}}=-\frac{\bar{E}_{\bar{u}}}{2 \bar{E}} l_{\bar{u}}-\bar{E} l .
$$

Hence, for the Lawson torus $M_{\alpha}$, the functions $a$ and $b$ in formulas (2-4) are $a=0$, $b=\alpha=$ const, and $\bar{E}=\bar{E}(\bar{u})$. The circles on $\mu_{\alpha}$ are the parametric $\bar{u}$-lines.

If we change the isothermal parameters $(\bar{u}, \bar{v})$ to isothermal parameters $(u, v)$ via

$$
u=(\bar{u}+\bar{v}) / \sqrt{2} \quad \text { and } \quad v=(\bar{u}-\bar{v}) / \sqrt{2},
$$

then $\widetilde{E}(u, v)=\left\langle l_{u}, l_{u}\right\rangle=\left\langle l_{v}, l_{v}\right\rangle=\widetilde{G}(u, v)$, and $l(u, v)$ satisfies (2-4) with $a=\alpha$, $b=0$ and $\widetilde{E}$ instead of $E$.

With respect to $(u, v)$, the surface $\mathcal{M}_{\alpha}$ is parametrized by principal lines, that is, the shape operator corresponding to the normal vector field $n(u, v)$ is in diagonal form. The circles on $M_{\alpha}$ are bisectrices of the principal lines.

Now we shall find all minimal surfaces in $S^{3}$ that are one-parameter families of circles crossing the principal lines at a constant angle. We call these surfaces generalized tori in $S^{3}$. They generalize the Lawson tori.

Proposition 2.1. Suppose $M^{2}$ is a minimal surface in $S^{3}$ with nonconstant Gauss curvature. Then $M^{2}$ can locally be parametrized by principal lines, and the new parameters are isothermal.

Proof. Let $M^{2}: l=l(u, v)$ for $(u, v) \in \mathscr{D}$ be a minimal surface in $S^{3}$ parametrized by isothermal parameters. Then the derivative formulas (2-4) of $M^{2}$ hold, and the functions $a(u, v)$ and $b(u, v)$ are harmonic functions satisfying the CauchyRiemann conditions. In case of $b(u, v) \equiv 0$, the parameters $(u, v)$ are principal. Let $b\left(u_{0}, v_{0}\right) \neq 0$ for $\left(u_{0}, v_{0}\right) \in \mathscr{D}$. Then there exists $\mathscr{D}_{0} \subset \mathscr{D}$ such that $b(u, v) \neq 0$ for all $(u, v) \in \mathscr{D}_{0}$. We shall prove that there exist isothermal parameters $(x, y)$ such that $\bar{b}(x, y)=\left\langle l_{x y}, n\right\rangle=0$. If $x=x(u, v)$ and $y=y(u, v)$ is a holomorphic change of the parameters (so that $x(u, v)$ and $y(u, v)$ satisfy the Cauchy-Riemann conditions), then $\bar{b}(x, y)=2 a u_{x} u_{y}-b\left(u_{x}^{2}-u_{y}^{2}\right)$. Hence,

$$
\bar{b}=0 \quad \text { if and only if } \quad b\left(\frac{u_{x}}{u_{y}}\right)^{2}-2 a\left(\frac{u_{x}}{u_{y}}\right)-b=0 .
$$

From the inverse change of parameters, using the Cauchy-Riemann conditions we have $x_{u}=u_{x} /\left(u_{x}^{2}+u_{y}^{2}\right)$ and $x_{v}=-u_{y} /\left(u_{x}^{2}+u_{y}^{2}\right)$, and hence we obtain that $\bar{b}=0$ if and only if

$$
b\left(\frac{x_{u}}{x_{v}}\right)^{2}+2 a\left(\frac{x_{u}}{x_{v}}\right)-b=0,
$$

that is, $x_{u} / x_{v}=\left(-a \pm \sqrt{a^{2}+b^{2}}\right) / b$. We write $\beta(u, v)=\left(-a+\sqrt{a^{2}+b^{2}}\right) / b$ and $\gamma(u, v)=\left(-a-\sqrt{a^{2}+b^{2}}\right) / b$. 
Now let us consider the equations

$$
\frac{d v}{d u}=\beta(u, v) \quad \text { and } \quad \frac{d v}{d u}=\gamma(u, v) .
$$

For each point $\left(u_{0}, v_{0}\right) \in \mathscr{D}_{0}$, there exist $\mathscr{D}_{1} \subset \mathscr{D}_{0}$ and functions $\Phi(u, v) \neq 0$ and $\Psi(u, v) \neq 0$ in $\mathscr{D}_{1}$, such that the integral curves of the first equation in (2-11) are given by $\Phi(u, v)=$ const, while the integral curves of the second equation in (2-11) are $\Psi(u, v)=$ const. Hence,

$$
\Phi_{u}=-\beta \Phi_{v} \quad \text { and } \quad \Psi_{u}=-\gamma \Psi_{v} .
$$

We consider the following smooth change of parameters:

$$
x=\Phi(u, v) \quad \text { and } \quad y=\Psi(u, v) \quad \text { for }(u, v) \in \mathscr{D}_{1} .
$$

When $(u, v)$ ranges over in $\mathscr{D}_{1}$, the parameters $(x, y)$ describe a domain $\overline{\mathscr{D}} \subset \mathbb{R}^{2}$. Now $x_{u}=\Phi_{u}, \quad x_{v}=\Phi_{v}, \quad y_{u}=\Psi_{u}$ and $y_{v}=\Psi_{v}$. Using $\beta \gamma=-1$ and equalities (2-12), we get $\left\langle l_{x}, l_{y}\right\rangle=0$, that is, the parametrization $(x, y)$ is orthogonal. We shall prove that this parametrization is isothermal. With respect to the new parameters, the coefficients of the first fundamental form are $E=\left\langle l_{x}, l_{x}\right\rangle, F=\left\langle l_{x}, l_{y}\right\rangle=0$, and $G=\left\langle l_{y}, l_{y}\right\rangle$. Then for the surface $M^{2}$, we have the derivative formulas

$$
\left\{\begin{aligned}
l_{x x} & =\frac{E_{x}}{2 E} l_{x}-\frac{E_{y}}{2 E} l_{y}-E l+a n, \\
l_{x y} & =\frac{E_{y}}{2 E} l_{x}+\frac{G_{x}}{2 G} l_{y}, \\
l_{y y} & =-\frac{G_{x}}{2 G} l_{x}+\frac{G_{y}}{2 G} l_{y}-G l-a n, \\
n_{x} & =-\frac{a}{E} l_{x} \text { and } n_{y}=\frac{a}{G} l_{y} .
\end{aligned}\right.
$$

Taking into account the second fundamental form of $M^{2}$ as a surface in $\mathbb{R}^{4}$, from (2-14) we calculate that the Gauss curvature $K$ is given by

$$
K=1-\frac{a^{2}}{E G} .
$$

Using $n_{x y}=n_{y x}, l_{x x y}=l_{x y x}$, and $l_{x y y}=l_{y y x}$, from (2-14) and (2-15) we obtain

$$
\begin{aligned}
& a_{x}=0, \quad a_{y}=0, \quad(E-G) E_{y}=0, \quad(E-G) G_{x}=0, \\
& \frac{E_{y y}}{2 E}+\frac{G_{x x}}{2 G}-\frac{3 E_{y}^{2}}{4 E^{2}}-\frac{G_{x}^{2}}{4 G^{2}}-\frac{E_{x} G_{x}-E_{y} G_{y}}{4 E G}+E-\frac{a^{2}}{G}=0, \\
& \frac{E_{y y}}{2 E}+\frac{G_{x x}}{2 G}-\frac{3 G_{x}^{2}}{4 G^{2}}-\frac{E_{y}^{2}}{4 E^{2}}+\frac{E_{x} G_{x}-E_{y} G_{y}}{4 E G}+G-\frac{a^{2}}{E}=0 .
\end{aligned}
$$


If we assume that $E\left(x_{0}, y_{0}\right)-G\left(x_{0}, y_{0}\right) \neq 0$ at some point $\left(x_{0}, y_{0}\right) \in \overline{\mathscr{D}}$, and hence $E-G \neq 0$ in a neighborhood $\overline{\mathscr{D}}_{0} \subset \overline{\mathscr{D}}$ of $\left(x_{0}, y_{0}\right)$, then from (2-17) we get $E_{y}=G_{x}=0$ in $\overline{\mathscr{D}}_{0}$. Now equalities (2-16), (2-17), and (2-18) imply that $K=0$ in $\overline{\mathscr{D}}_{0}$, which contradicts the assumption in the theorem. Hence, $E-G \equiv 0$ in $\overline{\mathscr{D}}$. Consequently, the parameters $(x, y)$ defined by change (2-13) are principal.

Now let $M^{2}: l=l(u, v)$ be a minimal surface in $S^{3}$ parametrized locally by isothermal principal parameters, that is, $b=0$. Using $b_{u}=a_{v}$ and $b_{v}=-a_{u}$, we get $a=$ const. Without loss of generality, we assume that $a=1$ (if $a \neq 1$, we multiply the parameters by $\sqrt{|a|}$ ). Hence the derivative formulas (2-4) and (2-5) hold with $a=1$ and $b=0$.

Theorem 2.2. Suppose $M^{2}$ is a minimal surface in $S^{3}$ with nonconstant Gauss curvature. If on $M^{2}$ there exists a family of circles crossing the principal lines at a constant angle $\theta$, then the circles are either principal lines $(\theta=0$ or $\theta=\pi / 2)$ or bisectrices of the principal lines $(\theta=\pi / 4$ or $\theta=3 \pi / 4)$.

Proof. Let $M^{2}: l=l(u, v)$ be parametrized locally by principal parameters. Suppose that on $M^{2}$ there exists a family of circles crossing the principal lines at a constant angle $\theta$. Let

$$
\begin{aligned}
& x=\cos \theta u+\sin \theta v, \\
& y=-\sin \theta u+\cos \theta v, \quad \theta=\text { const } \quad \text { for } \theta \in[0 ; 2 \pi) .
\end{aligned}
$$

Then from (2-19) we get

$$
E_{x}=\cos \theta E_{u}+\sin \theta E_{v} \text { and } E_{y}=-\sin \theta E_{u}+\cos \theta E_{v} .
$$

Using (2-4) with $a=1, \quad b=0$, and (2-20), we calculate

$$
\begin{aligned}
& l_{x x}=\frac{E_{x}}{2 E} l_{x}-\frac{E_{y}}{2 E} l_{y}-E l+\cos 2 \theta n, \\
& l_{x y}=\frac{E_{y}}{2 E} l_{x}+\frac{E_{x}}{2 E} l_{y}-\sin 2 \theta n, \\
& l_{y y}=-\frac{E_{x}}{2 E} l_{x}+\frac{E_{y}}{2 E} l_{y}-E l-\cos 2 \theta n .
\end{aligned}
$$

Let us write $a=\cos 2 \theta$ and $b=\sin 2 \theta(a, b-$ const $)$.

Consider an arbitrary $x$-line $c: l(x)=l\left(x, y_{0}\right)$ for constant $y_{0}$. It is a circle if and only if its Frenet curvatures are $\varkappa=$ const and $\tau=\sigma=0$. Using (2-21) we calculate the tangent vector $t_{c}$ and the principal normal vector $n_{c}$ of $c$ :

$$
t_{c}=\frac{l_{x}}{\sqrt{E}}, \quad n_{c}=\frac{1}{\varkappa}\left(-\frac{E_{y}}{2 E^{2}} l_{y}-l+\frac{a}{E} n\right), \quad \text { where } \varkappa^{2}=\frac{E_{y}^{2}}{4 E^{3}}+1+\frac{a^{2}}{E^{2}} .
$$


The derivatives of $t_{c}$ and $n_{c}$ are

$$
\begin{aligned}
t_{c}^{\prime}=\varkappa n_{c} & \\
n_{c}^{\prime}=- & \varkappa t_{c}-\left(\left(\frac{1}{\varkappa}\right)^{\prime} \frac{E_{y}}{2 E^{2}}+\frac{1}{\varkappa}\left(\left(\frac{E_{y}}{2 E^{2}}\right)_{x}+\frac{E_{x} E_{y}}{4 E^{3}}+\frac{a b}{E^{2}}\right)\right) \frac{l_{y}}{\sqrt{E}} \\
& \quad-\left(\frac{1}{\varkappa}\right)^{\prime} \frac{l}{\sqrt{E}}+\left(\left(\frac{1}{\varkappa}\right)^{\prime} \frac{a}{E}+\frac{1}{\varkappa}\left(-b \frac{E_{y}}{2 E^{2}}+\left(\frac{a}{E}\right)_{x}\right)\right) \frac{n}{\sqrt{E}} .
\end{aligned}
$$

From (2-22) it follows that $c$ is a circle if and only if

which is equivalent to

$$
\varkappa=\text { const, } \quad \begin{aligned}
\left(\frac{E_{y}}{2 E^{2}}\right)_{x}+\frac{E_{x} E_{y}}{4 E^{3}}+\frac{a b}{E^{2}} & =0, \\
\left(\frac{a}{E}\right)_{x}-b \frac{E_{y}}{2 E^{2}} & =0,
\end{aligned}
$$

$$
\begin{aligned}
2 a E_{x}+b E_{y} & =0, \\
2 E E_{x y}-3 E_{x} E_{y}+4 a b E & =0 .
\end{aligned}
$$

Analogously, the $y$-lines are circles if and only if

$$
\begin{aligned}
b E_{x}-2 a E_{y} & =0, \\
2 E E_{x y}-3 E_{x} E_{y}-4 a b E & =0 .
\end{aligned}
$$

From (2-20), we calculate

$$
E_{x y}=-\sin \theta \cos \theta E_{u u}+\cos 2 \theta E_{u v}+\sin \theta \cos \theta E_{v v} .
$$

From the first equality of (2-23), using (2-20), we get $\cos ^{3} \theta E_{u}-\sin ^{3} \theta E_{v}=0$. All solutions of this equation are given by

$$
E=\varphi\left(\sin ^{3} \theta u+\cos ^{3} \theta v\right)
$$

for an arbitrary smooth function $\varphi$. Hence, $E_{u}=\sin ^{3} \theta \varphi^{\prime}, E_{v}=\cos ^{3} \theta \varphi^{\prime}$, $E_{u u}=\sin ^{6} \theta \varphi^{\prime \prime}, \quad E_{v v}=\cos ^{6} \theta \varphi^{\prime \prime}$, and $E_{u v}=\sin ^{3} \theta \cos ^{3} \theta \varphi^{\prime \prime}$. From the second equality of (2-23), we obtain $\sin 2 \theta \cos 2 \theta\left(\varphi \varphi^{\prime \prime}-\frac{3}{2} \varphi^{\prime 2}-4 \varphi\right)=0$ using (2-25) and (2-26). Consequently, the $x$-lines are circles if and only if

$$
\begin{aligned}
E & =\varphi\left(\sin ^{3} \theta u+\cos ^{3} \theta v\right), \\
0 & =\sin 2 \theta \cos 2 \theta\left(\varphi \varphi^{\prime \prime}-\frac{3}{2} \varphi^{\prime 2}-4 \varphi\right) .
\end{aligned}
$$

Analogously, using (2-24) we obtain that the $y$-lines are circles if and only if

$$
\begin{aligned}
E & =\varphi\left(\cos ^{3} \theta u-\sin ^{3} \theta v\right), \\
0 & =\sin 2 \theta \cos 2 \theta\left(\varphi \varphi^{\prime \prime}-\frac{3}{2} \varphi^{\prime 2}-4 \varphi\right) .
\end{aligned}
$$

Thus the condition that the $x$-lines (or $y$-lines) be circles leads to the cases 
(I) $\sin 2 \theta=0$, that is, $\theta=0$ or $\theta=\pi / 2$.

This case corresponds to $x=u, y=v$ or $x=v, y=-u$. From (2-27) and (2-28) we obtain that the $u$-lines are circles if and only if $E=\varphi(v)$, and the $v$-lines are circles if and only if $E=\varphi(u)$. In this case, one of the families of principal lines is a family of circles.

(II) $\cos 2 \theta=0$, that is, $\theta=\pi / 4$ or $\theta=3 \pi / 4$.

This case corresponds to $x=(u+v) / \sqrt{2}, y=(-u+v) / \sqrt{2}$ or $x=(-u+v) / \sqrt{2}$, $y=-(u+v) / \sqrt{2}$. From (2-27) and (2-28) we obtain that the $x$-lines are circles if and only if $E=\varphi(x)$, and the $y$-lines are circles if and only if $E=\varphi(y)$. In this case one of the families of bisectrices of the principal lines is a family of circles.

(III) $\varphi \varphi^{\prime \prime}-\frac{3}{2} \varphi^{\prime 2}-4 \varphi=0$ and $\sin 2 \theta \cos 2 \theta \neq 0$.

We shall prove that this case is not possible. Since $E>0$, we have $E=\varphi(\tau)=e^{z(\tau)}$ for some function $z=z(\tau)$ with $\tau=\sin ^{3} \theta u+\cos ^{3} \theta v$ (or $\tau=\cos ^{3} \theta u-\sin ^{3} \theta v$ ). Moreover, $E \neq$ const, that is, $z^{\prime}(\tau) \neq 0$. The equality $\varphi \varphi^{\prime \prime}-\frac{3}{2} \varphi^{\prime 2}-4 \varphi=0$ implies

$$
z^{\prime \prime}-\frac{1}{2} z^{\prime 2}-4 e^{-z}=0 .
$$

On the other hand, using identity (2-6), we obtain

$$
\left(\cos ^{6} \theta+\sin ^{6} \theta\right) z^{\prime \prime}+4 \sinh z=0 .
$$

Let us write $\lambda=\cos ^{6} \theta+\sin ^{6} \theta=$ const. Multiplying (2-30) by $z^{\prime}$ and integrating, we get

$$
\frac{1}{2} \lambda z^{\prime 2}(\tau)+4 \cosh z(\tau)=\frac{1}{2} \lambda z^{\prime 2}(0)+4 \cosh z(0) .
$$

Equalities (2-29) and (2-30) imply

$$
4 \sinh z+\frac{1}{2} \lambda z^{\prime 2}+4 \lambda e^{-z}=0 .
$$

Using the last two equalities, we obtain

$$
4(1-\lambda) e^{-z}=\frac{1}{2} \lambda z^{\prime 2}(0)+4 \cosh z(0)=\text { const } .
$$

Since $1-\lambda=3 \sin ^{2} \theta \cos ^{2} \theta \neq 0$, we get $e^{-z(\tau)}=$ const, that is, $z(\tau)=$ const, which contradicts the condition $z^{\prime}(\tau) \neq 0$, that is, $E \neq$ const.

From Theorem 2.2 it follows that there are only two types of generalized tori in $S^{3}$ : the first are those such that one of the families of bisectrices of the principal lines is a family of circles (such surfaces are generalized tori of the first type); the second are those such that one of the families of principal lines is a family of circles (these are the generalized tori of the second type).

Theorem 2.3. Let $M^{2}$ be a generalized torus of the first type with nonconstant Gauss curvature. Then $M^{2}$ is a Lawson torus $M_{\alpha}$ for some positive $\alpha$ with $\alpha \neq 1$. 
Proof. Let $M^{2}$ be a generalized torus of the first type with nonconstant Gauss curvature. In this case the derivative formulas (2-4) hold with $a=0, b=1$, and $E=E(u)$ (or $E=E(v))$. We consider only the first case, in which $E=E(u)$ and $E_{u} \neq 0$. The second one can be investigated analogously. The derivative formulas in this case look like

$$
\begin{array}{rlrl}
l_{u u} & =\frac{E_{u}}{2 E} l_{u}-E l, & n_{u}=-\frac{1}{E} l_{v}, \\
l_{u v}=\frac{E_{u}}{2 E} l_{v}+n, & n_{v}=-\frac{1}{E} l_{u}, \\
l_{v v}=-\frac{E_{u}}{2 E} l_{u}-E l . &
\end{array}
$$

We shall prove that the parametric $u$-lines are great circles. Consider for constant $v_{0}$ the $u$-line $c: c(u)=l\left(u, v_{0}\right)$. From (2-31) it follows that $\dot{c}=l_{u}$, and the tangent vector $t_{c}$ of $c$ is $t_{c}=c^{\prime}=\dot{c} / \dot{s}=l_{u} / \sqrt{E}$. We calculate

$$
t_{c}^{\prime}=\frac{\dot{t}_{c}}{\dot{s}}=\frac{1}{\sqrt{E}}\left(\frac{l_{u u}}{\sqrt{E}}-\frac{E_{u}}{2 E \sqrt{E}} l_{u}\right)=-l .
$$

Hence the curvature $\varkappa$ of $c$ is $\varkappa=1$, so $c$ is a great circle. Consequently, $M^{2}$ is a oneparameter family of great circles. According to [Lawson 1970, Proposition 7.2], $M^{2}$ is an open submanifold of $M_{\alpha}$ for some positive $\alpha$ with $\alpha \neq 1$.

Now we consider a generalized torus of the second type with nonconstant Gauss curvature. In this case the derivative formulas (2-4) hold with $a=1, b=0$, and $E=E(u)$ (or $E=E(v))$. We consider the case $E=E(u)$ and $E_{u} \neq 0$, so that the parametric $v$-lines are circles. We prove that the different $v$-lines are circles with different radii.

Now the derivative formulas are

$$
\begin{array}{rlrl}
l_{u u} & =\frac{E_{u}}{2 E} l_{u}-E l+n, & & n_{u}=-\frac{1}{E} l_{u}, \\
l_{u v} & =\frac{E_{u}}{2 E} l_{v}, & n_{v}=\frac{1}{E} l_{v}, \\
l_{v v} & =-\frac{E_{u}}{2 E} l_{u}-E l-n . &
\end{array}
$$

For $u_{0}=$ const, we consider the $v$-line $c: c(v)=l\left(u_{0}, v\right)$. As in the proof of Theorem 2.3, from (2-32) we calculate the curvature $\varkappa\left(u_{0}\right)$ of $c$ to be

$$
\varkappa\left(u_{0}\right)=\sqrt{1+\frac{E_{u}^{2}\left(u_{0}\right)}{4 E^{3}\left(u_{0}\right)}+\frac{1}{E^{2}\left(u_{0}\right)}} .
$$


For different values of the constant $u_{0}$, the curvatures $\varkappa\left(u_{0}\right)$ are different. We note that $\varkappa\left(u_{0}\right)>1$, that is, the circles are not great ones. Therefore, the generalized tori of the second type differ from the Lawson tori.

Since $E(u)>0$, we write $E(u)$ in the form $E(u)=e^{z(u)}$ for $z(u) \neq$ const. Then the system (2-32) is rewritten in the form

$$
\begin{aligned}
l_{u u}-\frac{1}{2} z^{\prime}(u) l_{u}+e^{z(u)} l-n & =0, & & n_{u}+e^{-z(u)} l_{u}=0, \\
l_{u v}-\frac{1}{2} z^{\prime}(u) l_{v} & =0, & & n_{v}-e^{-z(u)} l_{v}=0, \\
l_{v v}+\frac{1}{2} z^{\prime}(u) l_{u}+e^{z(u)} l+n & =0 . & &
\end{aligned}
$$

We look for classical solutions of the system (2-33) for the vector-valued functions $l(u, v), l_{u}(u, v), l_{v}(u, v)$ and $n(u, v)$ in a neighborhood of the origin under the initial conditions

$$
\begin{aligned}
l(0,0) & =e_{1}, & l_{v}(0,0) & =e^{s / 2} e_{3}=\sqrt{E(0)} e_{3}, \\
l_{u}(0,0) & =e^{s / 2} e_{2}=\sqrt{E(0)} e_{2}, & n(0,0) & =e_{4},
\end{aligned}
$$

where $\left\{e_{1}, e_{2}, e_{3}, e_{4}\right\}$ is the standard orthonormal basis in $\mathbb{R}^{4}$, and $s=$ const $=$ $z(0)=\ln E(0)$.

Since the function $E(u)$ satisfies identity (2-6) with $a=1$ and $b=0$, it follows that $z(u)$ is the solution of the ordinary differential equation

$$
z^{\prime \prime}(u)+4 \sinh z(u)=0, \quad z(0)=s, \quad z^{\prime}(0)=2 t,
$$

where $s$ and $t$ are arbitrary constants.

To find $z(u)$ explicitly, we note that the identity (2-6) holds for an arbitrary minimal surface in $S^{3}$ parametrized by isothermal parameters. So let us consider again the Lawson torus $M_{\alpha}$ defined by (2-9). We change the parameters $(x, y)$ by new parameters $(u, v)$ via

$$
u=h(x)=\sqrt{\alpha} \int_{0}^{x} \frac{1}{\sqrt{\alpha^{2} \cos ^{2} \tau+\sin ^{2} \tau}} d \tau, \quad v=\sqrt{\alpha} y,
$$

and denote by $h^{-1}$ the inverse function of $h$. Then we obtain an isothermal parametrization of $\mu_{\alpha}$, and the function

$$
E(u)=e^{z(u)}=\frac{\alpha^{2} \cos ^{2} h^{-1}(u)+\sin ^{2} h^{-1}(u)}{\alpha}
$$

satisfies (2-6) with $a=0$ and $b=1$. Hence the function

$$
z(u)=\ln \frac{\alpha^{2} \cos ^{2} h^{-1}(u)+\sin ^{2} h^{-1}(u)}{\alpha}
$$


is a solution of (2-35) with $t=0$ and $s=\ln \alpha$ for $\alpha>0, \alpha \neq 1$. (It can be calculated directly that the function $z(u)$ defined by (2-37) satisfies (2-35) with $z(0)=\ln \alpha$ for $\alpha>0, \alpha \neq 1$ and $z^{\prime}(0)=0$.)

We will prove that every solution $\tilde{z}(u)$ of (2-35) with arbitrary $t$ and $s$ can be obtained from (2-37) by the formula $\tilde{z}(u)=z\left(u+u_{0}\right)$ for a suitable choice of constants $u_{0}$ and $\alpha$. Since (2-35) is an autonomous equation and $z(u)$ is its solution, $\tilde{z}(u)=z\left(u+u_{0}\right)$ is also a solution of this equation. Therefore we have to check only the initial conditions. Let $x_{0}=h^{-1}\left(u_{0}\right)$. Simple calculations give us the equalities

$$
\begin{gathered}
\tilde{z}(0)=z\left(u_{0}\right)=\ln \frac{\alpha^{2} \cos ^{2} x_{0}+\sin ^{2} x_{0}}{\alpha}=s, \\
\tilde{z}^{\prime}(0)=z^{\prime}\left(u_{0}\right)=\frac{\left(1-\alpha^{2}\right) \sin 2 x_{0}}{\sqrt{\alpha} \sqrt{\alpha^{2} \cos ^{2} x_{0}+\sin ^{2} x_{0}}}=2 t,
\end{gathered}
$$

which imply

$$
\begin{aligned}
\sin 2 x_{0} & =\frac{2 \alpha t e^{s / 2}}{1-\alpha^{2}}, \\
\cos 2 x_{0} & =\frac{1+\alpha^{2}-2 \alpha e^{s}}{1-\alpha^{2}} .
\end{aligned}
$$

Using $\sin ^{2} 2 x_{0}+\cos ^{2} 2 x_{0}=1$, we see that $\alpha$ satisfies

$$
e^{s} \alpha^{2}-\left(1+e^{2 s}+t^{2} e^{s}\right) \alpha+e^{s}=0
$$

whose positive solutions are

$$
\alpha=\frac{1+e^{2 s}+t^{2} e^{s} \pm \sqrt{\left(1+e^{2 s}+t^{2} e^{s}\right)^{2}-4 e^{2 s}}}{2 e^{s}} .
$$

For this choice of $\alpha$ and

$$
u_{0}=h\left(x_{0}\right)=\frac{1}{2} \arctan \frac{2 \alpha t e^{s / 2}}{1+\alpha^{2}-2 \alpha e^{s}},
$$

the initial conditions are satisfied.

Note that all solutions of (2-35) are periodic with period $\omega=h(\pi)$.

To simplify system (2-33), we change the vector function $l(u, v)$ with vector function $L(u, v)$ determined by

$$
l(u, v)=e^{z(u) / 2} L(u, v) .
$$


We get the system

$$
\begin{aligned}
& 0=L_{u u}(u, v)+\frac{1}{2} z^{\prime}(u) L_{u}(u, v)+e^{-z(u)} L(u, v)-e^{-z(u) / 2} n, \\
& 0=L_{u v}(u, v), \\
& 0=L_{v v}(u, v)+\frac{1}{2} z^{\prime}(u) L_{u}(u, v)+\left(\left(\frac{1}{2} z^{\prime}(u)\right)^{2}+e^{z(u)}\right) L(u, v)+e^{-z(u) / 2} n, \\
& 0=n_{u}+e^{-z(u) / 2}\left(L_{u}+\frac{1}{2} z^{\prime}(u) L\right), \\
& 0=n_{v}-e^{-z(u) / 2} L_{v} .
\end{aligned}
$$

The initial conditions (2-34) for $l(u, v)$ imply the following initial conditions for $L(u, v)$ :

$$
\begin{aligned}
L(0,0) & =e^{-s / 2} e_{1}, & L_{v}(0,0) & =e_{3}, \\
L_{u}(0,0) & =-t e^{-s / 2} e_{1}+e_{2}, & n(0,0) & =e_{4} .
\end{aligned}
$$

From the second equality in (2-39) it follows that $L(u, v)=f(u)+g(v)$, where $f(u)$ and $g(v)$ are vector functions satisfying the system

$$
\begin{aligned}
0 & =f^{\prime \prime}(u)+\frac{1}{2} z^{\prime}(u) f^{\prime}(u)+e^{-z(u)}(f(u)+g(v))-e^{-z(u) / 2} n, \\
0 & =g^{\prime \prime}(v)+\frac{1}{2} z^{\prime}(u) f^{\prime}(u)+\left(\left(\frac{1}{2} z^{\prime}(u)\right)^{2}+e^{z(u)}\right)(f(u)+g(v))+e^{-z(u) / 2} n, \\
0 & =n_{u}+e^{-z(u) / 2}\left(f^{\prime}(u)+\frac{1}{2} z^{\prime}(u)(f(u)+g(v))\right), \\
0 & =n_{v}-e^{-z(u) / 2} g^{\prime}(v)=0, \\
f^{\prime}(0) & =-t e^{-s / 2} e_{1}+e_{2}, \quad g^{\prime}(0)=e_{3}, \quad f(0)+g(0)=e^{-s / 2} e_{1} .
\end{aligned}
$$

Without loss of generality we assume that $g(0)=0$ and $f(0)=e^{-s / 2} e_{1}$.

Let us fix $u=0$ in the fourth equality of (2-40). Then after integration we get $n(0, v)=e^{-s / 2} g(v)+e_{4}$. Now using the second equality of (2-40), we see that $g(v)$ satisfies the initial value problem

$$
\begin{aligned}
& g^{\prime \prime}(v)+\left(t^{2}+2 \cosh s\right) g(v)=-e^{s / 2} e_{1}-t e_{2}-e^{-s / 2} e_{4}, \\
& g(0)=0, \quad g^{\prime}(0)=e_{3} .
\end{aligned}
$$

Simple computations give us

$$
\begin{array}{r}
g(v)=\frac{1}{t^{2}+2 \cosh s}\left(\cos \sqrt{t^{2}+2 \cosh s} v-1\right)\left(e^{s / 2} e_{1}+t e_{2}+e^{-s / 2} e_{4}\right) \\
+\frac{1}{\sqrt{t^{2}+2 \cosh s}} \sin \sqrt{t^{2}+2 \cosh s} v e_{3} .
\end{array}
$$

Now, multiplying (2-35) with $z^{\prime}(u)$ and integrating from 0 to $u$, we get that $z^{\prime}(u)$ satisfies the equality

$$
\left(z^{\prime}(u)\right)^{2}+8 \cosh z(u)=4 t^{2}+8 \cosh s .
$$


Using the first and second equalities of (2-40), (2-41), and (2-42), and setting $v=0$, we see that $f(u)$ satisfies the initial value problem

$$
\begin{aligned}
& f^{\prime \prime}(u)+z^{\prime}(u) f^{\prime}(u)+\left(t^{2}+2 \cosh s\right) f(u)=e^{s / 2} e_{1}+t e_{2}+e^{-s / 2} e_{4}, \\
& f(0)=e^{-s / 2} e_{1}, \quad f^{\prime}(0)=-t e^{-s / 2} e_{1}+e_{2} .
\end{aligned}
$$

Therefore the solution $f(u)$ of the system above can be written in the form

$$
f(u)=p(u)+\frac{1}{t^{2}+2 \cosh s}\left(e^{s / 2} e_{1}+t e_{2}+e^{-s / 2} e_{4}\right),
$$

where $p(u)$ is the unique solution of the linear homogeneous system

$$
\begin{aligned}
& p^{\prime \prime}(u)+z^{\prime}(u) p^{\prime}(u)+\left(t^{2}+2 \cosh s\right) p(u)=0, \\
& p(0)=\frac{1}{t^{2}+2 \cosh s}\left[e^{-s / 2}\left(t^{2}+e^{-s}\right) e_{1}-t e_{2}-e^{-s / 2} e_{4}\right], \quad p^{\prime}(0)=-t e^{-s / 2} e_{1}+e_{2} .
\end{aligned}
$$

By (2-38), the solution $l(u, v)$ of problem (2-33) under condition (2-34) is

$$
\begin{aligned}
l(u, v)=e^{z(u) / 2} p(u)+\frac{e^{z(u) / 2}}{t^{2}+2 \cosh s} \cos \sqrt{t^{2}+2 \cosh s} v\left(e^{s / 2} e_{1}+t e_{2}+e^{-s / 2} e_{4}\right) \\
+\frac{e^{z(u) / 2}}{\sqrt{t^{2}+2 \cosh s}} \sin \sqrt{t^{2}+2 \cosh s} v e_{3} .
\end{aligned}
$$

If we denote $\beta=\sqrt{t^{2}+2 \cosh s}$ (here $\beta$ is constant), then $l(u, v)$ is rewritten as

$$
l(u, v)=e^{z(u) / 2} p(u)+\frac{e^{z(u) / 2}}{\beta^{2}}\left(\cos \beta v\left(e^{s / 2} e_{1}+t e_{2}+e^{-s / 2} e_{4}\right)+\beta \sin \beta v e_{3}\right) .
$$

The function $z(u)$ is given explicitly by (2-37). Thus we have proved this:

Theorem 2.4. Let $M^{2}: l=l(u, v)$ be a generalized torus of the second type with nonconstant Gauss curvature. Then

$$
l(u, v)=e^{z(u) / 2} p(u)+\frac{e^{z(u) / 2}}{\beta^{2}}\left(\cos \beta v\left(e^{s / 2} e_{1}+t e_{2}+e^{-s / 2} e_{4}\right)+\beta \sin \beta v e_{3}\right),
$$

where $\beta=\sqrt{t^{2}+2 \cosh s}$ for arbitrary constants $s$ and $t$. The scalar function $z(u)$ is the solution of the initial value problem

$$
z^{\prime \prime}+4 \sinh z(u)=0, \quad z(0)=s, \quad z^{\prime}(0)=2 t,
$$

and is given explicitly by (2-37). The vector function $p(u)$ is a solution of the system

$$
\begin{aligned}
& p^{\prime \prime}(u)+z^{\prime}(u) p^{\prime}(u)+\beta^{2} p(u)=0, \\
& p(0)=\frac{1}{\beta^{2}}\left(e^{-s / 2}\left(t^{2}+e^{-s}\right) e_{1}-t e_{2}-e^{-s / 2} e_{4}\right), \quad p^{\prime}(0)=-t e^{-s / 2} e_{1}+e_{2},
\end{aligned}
$$

and $\left\{e_{1}, e_{2}, e_{3}, e_{4}\right\}$ is the standard orthonormal basis in $\mathbb{R}^{4}$. 


\section{Application to the theory of minimal foliated semisymmetric hypersurfaces}

We now relate the theory of minimal surfaces in $S^{3}$ to the theory of minimal foliated semisymmetric hypersurfaces in $\mathbb{R}^{4}$.

For an $n$-dimensional Riemannian manifold $\left(M^{n}, g\right)$, we denote by $T_{p} M^{n}$ the tangent space to $M^{n}$ at a point $p \in M^{n}$ and by $\mathfrak{X} M^{n}$ the algebra of all vector fields on $M^{n}$. The associated Levi-Civita connection of the metric $g$ is denoted by $\nabla$ and the Riemannian curvature tensor $R$ is defined by

$$
R(X, Y)=\left[\nabla_{X}, \nabla_{Y}\right]-\nabla_{[X, Y]} \text { for } X, Y \in \mathfrak{X} M^{n} .
$$

A semisymmetric space is a Riemannian manifold $\left(M^{n}, g\right)$ such that $R$ satisfies the identity $R(X, Y) \cdot R=0$ for all vector fields $X, Y \in \mathfrak{X} M^{n}$. (Here $R(X, Y)$ acts as a derivation on $R$ ).

According to the classification of Z. Szabó [1982], the main class of semisymmetric spaces is the class of all Riemannian manifolds foliated by Euclidean leaves of codimension two.

The foliated semisymmetric hypersurfaces in the Euclidean space $\mathbb{E}^{n+1}$ are the hypersurfaces of type number two, that is, those hypersurfaces whose second fundamental form has rank two everywhere. They are characterized by a second fundamental form $h=v_{1} \eta_{1} \otimes \eta_{1}+v_{2} \eta_{2} \otimes \eta_{2}$ for $v_{1} v_{2} \neq 0$, where $\eta_{1}$ and $\eta_{2}$ are unit oneforms, and $v_{1}$ and $v_{2}$ are functions on the hypersurface $M^{n}$. The Euclidean leaves of the foliation are the integral submanifolds of the distribution $\Delta_{0}$, determined by the one-forms $\eta_{1}$ and $\eta_{2}$, that is, $\Delta_{0}(p)=\left\{X \in T_{p} M^{n} \mid \eta_{1}(X)=0, \eta_{2}(X)=0\right\}$ for $p \in M^{n}$. A special class of foliated semisymmetric hypersurfaces is the class of ruled hypersurfaces.

A hypersurface $M^{n}$ of type number two is minimal if $v_{1}+v_{2}=0$.

Ganchev and Milousheva [2007b] have characterized the foliated semisymmetric hypersurfaces in $\mathbb{E}^{n+1}$ as follows.

Theorem 3.1. A hypersurface $M^{n}$ in Euclidean space $\mathbb{E}^{n+1}$ is locally a foliated semisymmetric hypersurface if and only if it is the envelope of a two-parameter family of hyperplanes in $\mathbb{E}^{n+1}$.

If we consider a foliated semisymmetric hypersurface as the envelope of a twoparameter family of hyperplanes, each such hypersurface is determined by a pair consisting of a unit vector-valued function $l(u, v)$ and a scalar function $r(u, v)$, both defined in a domain $\mathscr{D} \subset \mathbb{R}^{2}$.

Since the vector fields $l_{u}$ and $l_{v}$ are linearly independent, $l(u, v)$ determines a two-dimensional surface $M^{2}: l=l(u, v)$ for $(u, v) \in \mathscr{D}$ in $\mathbb{E}^{n+1}$. Without loss of generality, it can be assumed that the surface $M^{2}$ is parametrized locally by isothermal parameters, namely, $E=G$ and $F=0$. Then the generated foliated 
semisymmetric hypersurface $M^{n}$ is given in [Ganchev and Milousheva 2007a] by

$$
X\left(u, v, w^{\alpha}\right)=r l+\frac{r_{u}}{E} l_{u}+\frac{r_{v}}{E} l_{v}+w^{\alpha} b_{\alpha} \quad \text { for } \alpha=1, \ldots, n-2,
$$

where $(u, v) \in \mathscr{D}, w^{\alpha} \in \mathbb{R}$ for $\alpha=1, \ldots, n-2$ and $b_{1}(u, v), \ldots, b_{n-2}(u, v)$, where $(u, v) \in \mathscr{D}$ are $n-2$ mutually orthogonal unit vectors, orthogonal to $\operatorname{span}\left\{l, l_{u}, l_{v}\right\}$.

The minimal foliated semisymmetric hypersurfaces in $\mathbb{E}^{n+1}$ are characterized analytically in [Ganchev and Milousheva 2007a] as follows.

Theorem 3.2. Let $M^{n}$ be a hypersurface in $\mathbb{E}^{n+1}$ that is the envelope of a twoparameter family of hyperplanes and is determined by a unit vector-valued function $l(u, v)$, represented by isothermal parameters, and a scalar function $r(u, v)$. Then $M^{n}$ is minimal if and only if $l(u, v)$ and $r(u, v)$ satisfy

$$
\begin{aligned}
\Delta l(u, v)+2 E(u, v) l(u, v) & =0, \\
\Delta r(u, v)+2 E(u, v) r(u, v) & =0 .
\end{aligned}
$$

Hence, the minimal foliated semisymmetric hypersurfaces in $\mathbb{R}^{4}$ are generated by the solutions of system (2-3), that is, by the minimal surfaces in $S^{3}$.

Now we construct examples of minimal foliated semisymmetric hypersurfaces in $\mathbb{R}^{4}$ that are generated by some minimal surfaces in $S^{3}$.

The simplest example of a minimal surface in $S^{3}$ is the sphere $S^{2}=S^{3} \cap \mathbb{R}^{3}$. We assume that $\mathbb{R}^{3}$ is the subspace of $\mathbb{R}^{4}$ orthogonal to $e_{4}$, that is, $\mathbb{R}^{3}=\operatorname{span}\left\{e_{1}, e_{2}, e_{3}\right\}$. An isothermal parametrization of $S^{2}$ is given by

$$
S^{2}: l(u, v)=\frac{1}{\cosh u}(\cos v ; \sin v ; \sinh u ; 0) .
$$

A direct check shows that $E=\left\langle l_{u}, l_{u}\right\rangle=\left\langle l_{v}, l_{v}\right\rangle=1 / \cosh ^{2} u$ and $F=\left\langle l_{u}, l_{v}\right\rangle=0$, and obviously $l(u, v)$ satisfies the equality

$$
\Delta l(u, v)+\frac{2}{\cosh ^{2} u} l(u, v)=0 .
$$

The normal vector field $n(u, v)$ of $S^{2}$ is $n=e_{4}=(0 ; 0 ; 0 ; 1)$. By Theorem 3.2, the corresponding differential equation for the scalar function $r(u, v)$ is

$$
\Delta r(u, v)+\frac{2}{\cosh ^{2} u} r(u, v)=0 .
$$

Every solution $r(u, v)$ of (3-2) together with the sphere $S^{2}: l=l(u, v)$ generates a minimal foliated semisymmetric hypersurface in $\mathbb{R}^{4}$ according to formula (3-1).

One solution of (3-2) is

$$
r(u, v)=\left(v+\frac{1}{2} \pi\right) \tanh u .
$$


Now consider the minimal foliated semisymmetric hypersurface $M^{3}$ generated by $l(u, v)$ and this solution $r(u, v)$. Calculating $r_{u}, r_{v}, l_{u}, l_{v}$, and applying formula (3-1), we obtain $M^{3}: X(u, v, w)=\left(-\sinh u \sin v ; \sinh u \cos v ; v+\frac{1}{2} \pi ; w\right)$. After changing to the parameters $u^{1}=\sinh u$ and $t=v+\frac{1}{2} \pi$, we obtain the hypersurface

$$
M^{3}: X\left(u^{1}, t, w\right)=u^{1}\left(\cos t e_{1}+\sin t e_{2}\right)+t e_{3}+w e_{4} .
$$

The hypersurface $M^{3}$ whose radius vector $X=X\left(u^{1}, t, w\right)$ is determined by (3-3) is the generalized helicoidal ruled hypersurface obtained by G. Aumann [1981, Theorem 4]. It is also called a first type helicoid in $\mathbb{R}^{4}$ and is a generalization of the right helicoid in $\mathbb{R}^{3}$.

The next well-known example of a minimal surface in $S^{3}$ is the Clifford torus

$$
M: l(u, v)=(\cos u \cos v ; \cos u \sin v ; \sin u \cos v ; \sin u \sin v) .
$$

The normal vector field $n(u, v)$ of $\mathcal{M}$ is

$$
n(u, v)=(\sin u \sin v ;-\sin u \cos v ;-\cos u \sin v ; \cos u \cos v) .
$$

Since $E=\left\langle l_{u}, l_{u}\right\rangle=\left\langle l_{v}, l_{v}\right\rangle=1$, Theorem 3.2 says that the corresponding equation for the scalar function $r(u, v)$ is $\Delta r(u, v)+2 r(u, v)=0$. If we take the trivial solution $r(u, v)=0$, we obtain the minimal foliated semisymmetric hypersurface $M^{3}$ : $X(u, v, w)=w n(u, v)=w(\sin u \sin v ;-\sin u \cos v ;-\cos u \sin v ; \cos u \cos v)$.

After the change of parameters $u^{1}=-w \sin u, u^{2}=w \cos u$, and $t=v+\frac{1}{2} \pi$, we obtain the hypersurface

$$
M^{3}: X\left(u^{1}, u^{2}, t\right)=u^{1}\left(\cos t e_{1}+\sin t e_{2}\right)+u^{2}\left(\cos t e_{3}+\sin t e_{4}\right) .
$$

The hypersurface $M^{3}$ whose radius vector $X=X\left(u^{1}, u^{2}, t\right)$ is determined by (3-4) is the minimal ruled hypersurface obtained by G. Aumann [1981, Theorem 1], and is known as a second type helicoid. The helicoids of first and second type are the only minimal ruled hypersurfaces in $\mathbb{R}^{4}$ [Aumann 1981].

Thus we have shown that the first type helicoid is generated by the sphere $S^{2}$ in $S^{3}$, while the second type helicoid is generated by the Clifford torus.

Our scheme of constructing minimal foliated semisymmetric hypersurfaces in $\mathbb{R}^{4}$ can be applied to each minimal surface $M^{2}: l=l(u, v)$ in $S^{3}$ and each solution of the corresponding differential equation for the scalar function $r(u, v)$.

We illustrate how this construction can be applied to the generalized torus of the second type, given in Theorem 2.4, in the special case when $t=0$ and $s=\ln \alpha$ for positive $\alpha \neq 1$. Since the calculations are too long and complicated, we give only a short sketch. In this case the solution $l(u, v)$ is defined by

$$
l(u, v)=f(u)\left(p(u)+\frac{\alpha}{\alpha^{2}+1} \cos \beta v\left(\sqrt{\alpha} e_{1}+\frac{1}{\sqrt{\alpha}} e_{4}\right)+\sqrt{\frac{\alpha}{\alpha^{2}+1}} \sin \beta v e_{3}\right),
$$


where $f(u)=\sqrt{\left(\alpha^{2} \cos ^{2} h^{-1}(u)+\sin ^{2} h^{-1}(u)\right) / \alpha}, h^{-1}(u)$ is the inverse function of $h$ given in (2-36), $p(u)$ is the solution of system (2-43), and $\beta=\sqrt{\left(\alpha^{2}+1\right) / \alpha}$. As a solution of the corresponding differential equation for $r(u, v)$, we choose $r(u, v)=f(u) \sqrt{\alpha /\left(\alpha^{2}+1\right)} \sin \beta v$. We calculate $l_{u}(u, v)$ and $l_{v}(u, v)$, and using (2-33) and (2-41), we find the vector-valued function

$$
\begin{aligned}
& n(u, v)=\frac{1-\alpha^{2}}{\alpha\left(\alpha^{2}+1\right)}\left(e_{1}-\alpha e_{4}\right)+\frac{\alpha}{\left(\alpha^{2}+1\right) f(u)} \cos \beta v\left(\sqrt{\alpha} e_{1}+\frac{1}{\sqrt{\alpha}} e_{4}\right) \\
& +\sqrt{\frac{\alpha}{\alpha^{2}+1}} \frac{1}{f(u)} \sin \beta v e_{3}-\frac{p(u)}{f(u)}-\frac{1-\alpha^{2}}{\alpha} \int_{0}^{u} \frac{\sin 2 h^{-1}(s)}{f^{2}(s)} p(s) d s .
\end{aligned}
$$

Applying formula (3-1), we obtain the following minimal foliated semisymmetric hypersurface $M^{3}$ :

$$
\begin{aligned}
& X(u, v, w)= \frac{1-\alpha^{2}}{2 \sqrt{\alpha\left(\alpha^{2}+1\right)}} \frac{\sin 2 h^{-1}(u)}{f(u)} \sin \beta v p^{\prime}(u) \\
&+\sqrt{\frac{\alpha}{\alpha^{2}+1}} \frac{\alpha^{4} \cos ^{2} h^{-1}(u)+\sin ^{2} h^{-1}(u)}{\alpha^{2} f^{2}(u)} \sin \beta v p(u) \\
&-\left(\sqrt{\frac{\alpha}{\alpha^{2}+1}}\right)^{3} \frac{1}{2 f^{2}(u)} \sin 2 \beta v\left(\sqrt{\alpha} e_{1}+\frac{1}{\sqrt{\alpha}} e_{4}\right)+\left(1-\frac{\alpha}{\left(\alpha^{2}+1\right) f^{2}(u)} \sin ^{2} \beta v\right) e_{3} \\
&+w n(u, v) .
\end{aligned}
$$

Unfortunately, in this case we cannot write the hypersurface $M^{3}$ in terms of elementary functions as in the previous examples, because we cannot find an explicit solution $p(u)$ of linear system (2-43).

\section{References}

[Aumann 1981] G. Aumann, "Die Minimalhyperregelflächen”, Manuscripta Math. 34:2-3 (1981), 293-304. MR 82h:53007 Zbl 0452.53003

[Enneper 1869] A. Enneper, “Die cyklischen Flächen”, Z. Math. Phys. 14 (1869), 393-421. JFM 02.0585 .01

[Ganchev and Milousheva 2007a] G. Ganchev and V. Milousheva, "Analytical characterization of the minimal and bi-umbilical foliated semi-symmetric hypersurfaces in Euclidean space", $C$. $R$. Acad. Bulgare Sci. 60:6 (2007), 601-606. MR 2008h:53095 Zbl 1174.53030

[Ganchev and Milousheva 2007b] G. Ganchev and V. Milousheva, "A generation of foliated semisymmetric hypersurfaces in four-dimensional Euclidean space", Math. Balkanica (N.S.) 21:1-2 (2007), 97-111. MR 2008g:53067 Zbl 1147.53045

[Giaquinta et al. 1998a] M. Giaquinta, G. Modica, and J. Souček, Cartesian currents in the calculus of variations, I: Cartesian currents, Ergebnisse der Mathematik und ihrer Grenzgebiete (3) 37, Springer, Berlin, 1998. MR 1645086 Zbl 0914.49001

[Giaquinta et al. 1998b] M. Giaquinta, G. Modica, and J. Souček, Cartesian currents in the calculus of variations, II: Variational integrals, Ergebnisse der Mathematik und ihrer Grenzgebiete (3) 38, Springer, Berlin, 1998. MR 1645082 Zbl 0914.49002 
[Hildebrandt 1982] S. Hildebrandt, "Nonlinear elliptic systems and harmonic mappings", pp. 481615 in Proceedings of the 1980 Beijing Symposium on Differential Geometry and Differential Equations, vol. 1, edited by S. S. Chern and W.-T. Wu, Science Press/Gordon and Breach, Beijing/New York, 1982. MR 714341 Zbl 0515.58012

[Jost 1984] J. Jost, Harmonic maps between surfaces, Lecture Notes in Mathematics 1062, Springer, Berlin, 1984. MR 85j:58046 Zbl 0542.58002

[Jost 1991] J. Jost, Two-dimensional geometric variational problems, Wiley \& Sons, Chichester, 1991. MR 92h:58045 Zbl 0729.49001

[Lawson 1969] H. B. Lawson, Jr., "Local rigidity theorems for minimal hypersurfaces", Ann. of Math. (2) 89:1 (1969), 187-197. MR 38 \#6505 Zbl 0174.24901

[Lawson 1970] H. B. Lawson, Jr., "Complete minimal surfaces in $S^{3}$ ", Ann. of Math. (2) $92: 3$ (1970), 335-374. MR 42 \#5170 Zbl 0205.52001

[López 2001] R. López, "Cyclic surfaces of constant Gauss curvature", Houston J. Math. 27:4 (2001), 799-805. MR 2003a:53009 Zbl 1011.53006

[Nitsche 1989] J. C. C. Nitsche, "Cyclic surfaces of constant mean curvature", Nachr. Akad. Wiss. Göttingen Math.-Phys. Kl. II 1 (1989), 1-5. MR 90k:53012 Zbl 0714.53006

[Riemann 1868] B. Riemann, "Über die Fläche vom kleinsten Inhalt bei gegebener Begrenzung", Abh. Königl. Ges. Wissensch. Göttingen Mathem. Kl. 13 (1868), 329-333. Zbl JFM 01.0218.01

[Struwe 1988] M. Struwe, Plateau's problem and the calculus of variations, Mathematical Notes 35, Princeton University Press, Princeton, NJ, 1988. MR 90h:58016 Zbl 0694.49028

[Szabó 1982] Z. I. Szabó, "Structure theorems on Riemannian spaces satisfying $R(X, Y) \cdot R=0$, I: The local version”, J. Differential Geom. 17:4 (1982), 531-582. MR 84e:53060 Zbl 0508.53025

Received July 16, 2009. Revised November 27, 2009.

\section{Nikolai KuteV}

BULGARIAN ACADEMY OF SCIENCES

INSTITUTE OF MATHEMATICS AND INFORMATICS

ACAD. G. BONCHEV STR. BL. 8

1113 SOFIA

BULGARIA

kutev@math.bas.bg

VELICHKA MilousheVA

BULGARIAN ACADEMY OF SCIENCES

INSTITUTE OF MATHEMATICS AND INFORMATICS

ACAD. G. BONCHEV STR. BL. 8

1113 SOFIA

BULGARIA

and

L. Karavelov Higher School of Civil Engineering

175 SUHODOLSKA STR.

1373 SOFIA

BULGARIA

vmil@math.bas.bg 


\title{
PACIFIC JOURNAL OF MATHEMATICS
}

\author{
http://www.pjmath.org \\ Founded in 1951 by \\ E. F. Beckenbach (1906-1982) and F. Wolf (1904-1989)
}

\section{EDITORS}

V. S. Varadarajan (Managing Editor)

Department of Mathematics

University of California

Los Angeles, CA 90095-1555

pacific@math.ucla.edu

Vyjayanthi Chari

Department of Mathematics

University of California

Riverside, CA 92521-0135

chari@math.ucr.edu

Robert Finn

Department of Mathematics Stanford University

Stanford, CA 94305-2125

finn@math.stanford.edu

Kefeng Liu

Department of Mathematics

University of California

Los Angeles, CA 90095-1555

liu@math.ucla.edu
Darren Long

Department of Mathematics

University of California

Santa Barbara, CA 93106-3080

long@math.ucsb.edu

Jiang-Hua Lu

Department of Mathematics

The University of Hong Kong

Pokfulam Rd., Hong Kong jhlu@maths.hku.hk

Alexander Merkurjev

Department of Mathematics

University of California

Los Angeles, CA 90095-1555

merkurev@math.ucla.edu
Sorin Popa

Department of Mathematics University of California

Los Angeles, CA 90095-1555 popa@math.ucla.edu

Jie Qing

Department of Mathematics

University of California

Santa Cruz, CA 95064

qing@cats.ucsc.edu

Jonathan Rogawski

Department of Mathematics

University of California

Los Angeles, CA 90095-1555

jonr@math.ucla.edu

\section{PRODUCTION}

pacific@math.berkeley.edu

\begin{abstract}
Silvio Levy, Scientific Editor Matthew Cargo, Senior Production Editor
\end{abstract}
ACADEMIA SINICA, TAIPEI

CALIFORNIA INST. OF TECHNOLOGY

INST. DE MATEMÁTICA PURA E APLICADA

KEIO UNIVERSITY

MATH. SCIENCES RESEARCH INSTITUTE

NEW MEXICO STATE UNIV.

OREGON STATE UNIV.

\section{SUPPORTING INSTITUTIONS}

STANFORD UNIVERSITY
UNIV. OF BRITISH COLUMBIA
UNIV. OF CALIFORNIA, BERKELEY
UNIV. OF CALIFORNIA, DAVIS
UNIV. OF CALIFORNIA, LOS ANGELES
UNIV. OF CALIFORNIA, RIVERSIDE
UNIV. OF CALIFORNIA, SAN DIEGO
UNIV. OF CALIF., SANTA BARBARA

UNIV. OF CALIF., SANTA CRUZ

UNIV. OF MONTANA

UNIV. OF OREGON

UNIV. OF SOUTHERN CALIFORNIA

UNIV. OF UTAH

UNIV. OF WASHINGTON

WASHINGTON STATE UNIVERSITY

These supporting institutions contribute to the cost of publication of this Journal, but they are not owners or publishers and have no responsibility for its contents or policies.

See inside back cover or www.pjmath.org for submission instructions.

The subscription price for 2010 is US \$420/year for the electronic version, and \$485/year for print and electronic.

Subscriptions, requests for back issues from the last three years and changes of subscribers address should be sent to Pacific Journal of Mathematics, P.O. Box 4163, Berkeley, CA 94704-0163, U.S.A. Prior back issues are obtainable from Periodicals Service Company, 11 Main Street, Germantown, NY 12526-5635. The Pacific Journal of Mathematics is indexed by Mathematical Reviews, Zentralblatt MATH, PASCAL CNRS Index, Referativnyi Zhurnal, Current Mathematical Publications and the Science Citation Index.

The Pacific Journal of Mathematics (ISSN 0030-8730) at the University of California, c/o Department of Mathematics, 969 Evans Hall, Berkeley, CA 94720-3840, is published monthly except July and August. Periodical rate postage paid at Berkeley, CA 94704, and additional mailing offices. POSTMASTER: send address changes to Pacific Journal of Mathematics, P.O. Box 4163, Berkeley, CA 94704-0163.

PJM peer review and production are managed by EditFLOW ${ }^{\mathrm{TM}}$ from Mathematical Sciences Publishers.

PUBLISHED BY PACIFIC JOURNAL OF MATHEMATICS

at the University of California, Berkeley 94720-3840

A NON-PROFIT CORPORATION

Typeset in LATEX

Copyright $(\mathrm{C} 2010$ by Pacific Journal of Mathematics 


\section{PACIFIC JOURNAL OF MATHEMATICS}

Volume $248 \quad$ No. $2 \quad$ December 2010

Topological description of Riemannian foliations with dense leaves

Jesús A. ÁlVAREZ LóPEZ and Alberto CANDEL

The nonexistence of quasi-Einstein metrics

JEFFREY S. CASE

Twisted symmetric group actions

AKINARI HoSHI and MING-CHANG KANG

Optimal transportation and monotonic quantities on evolving manifolds

305

HONG HUANG

Hopf structures on the Hopf quiver $Q(\langle g\rangle, g)$

HuA-Lin HuANG, YU Ye and QING ZHaO

Minimal surfaces in $S^{3}$ foliated by circles

Nikolai KuteV and VelichKa Milousheva

Prealternative algebras and prealternative bialgebras

XIANG Ni and CHENGMING BAI

Some remarks about closed convex curves

Ke OU and Shengliang PAN

Orbit correspondences for real reductive dual pairs

SHU-YEN PAN

Graphs of bounded degree and the $p$-harmonic boundary

Michael J. Puls

Invariance of the BFV complex

FLORIAN SCHÄTZ

Some elliptic PDEs on Riemannian manifolds with boundary

YANNICK SIRE and ENRICO VALDINOCI

Representations of Lie superalgebras in prime characteristic, III 